\title{
CHARACTERIZATION OF CONDITIONAL EXPECTATION OPERATORS FOR BANACH-VALUED FUNCTIONS
}

\author{
D. LANDERS AND L. ROGGE
}

\begin{abstract}
Let $B$ be a Banach space. It is well known that for every probability space $(\Omega, Q, P)$ and every sub- $\sigma$-field $\mathbb{Q}_{0} \subset \mathbb{Q}$ there exists a conditional expectation operator $P^{a_{0}} X$ for $B$-valued $P$-integrable functions. This operator maps the space $L_{p}(\Omega, Q, P, B)$ into itself for each $p>1$. The operator is linear, idempotent, constant preserving and contractive in $L_{p}$. For $B=\mathbf{R}$ and $p \neq 2$ these conditions characterize a conditional expectation operator. It turns out that in general these properties characterize conditional expectation operators for Banach-valued functions only for $p=1$ and strictly convex Banach spaces.
\end{abstract}

Let $(\Omega, \mathcal{Q}, P)$ be a probability space, $\mathbb{Q}_{0} \subset \mathbb{Q}$ a $\sigma$-field and $1<p<\infty$. Let $B$ be a real Banach space with norm | | Consider the space $L_{p}(\Omega, Q, P, B)$ of all equivalence classes of $B$ strongly measurable functions $X: \Omega \rightarrow B$ such that $|X|^{p}$ is $P$-integrable. Then the conditional expectation operator

$$
T=P^{\mathbb{Q}_{0}}: L_{p}(\Omega, Q, P, B) \rightarrow L_{p}(\Omega, \mathcal{Q}, P, B)
$$

has the following properties (see [6, Proposition $\mathrm{V} 2-5]$ ):

(i) $T$ is linear, i.e. $T\left(\alpha X_{1}+\beta X_{2}\right)=\alpha T X_{1}+\beta T X_{2}$.

(ii) $T$ is idempotent, i.e. $T(T X)=T X$.

(iii) $T$ is constant preserving, i.e. $T b=b$ for $b \in B$.

(iv) $T$ is \|\|$_{p}$-contractive, i.e.

$$
\|T X\|_{p}:=\left(\int|T X|^{p} d P\right)^{1 / p}<\left(\int|X|^{p} d P\right)^{1 / p}=:\|X\|_{p} .
$$

For $B=\mathbf{R}$ and $p \neq 2$ these four conditions characterize the conditional expectation operators (see [6, Proposition I-2-14]). M. M. Rao (see [9, Theorem 2]) has tried to prove a corresponding result for more general Banach spaces. For a certain class of Banach spaces $B$ he has shown that each operator $T: L_{p}(\Omega, Q, P, B) \rightarrow$ $L_{p}(\Omega, Q, P, B)$ fulfilling (i)-(iv) is a conditional expectation operator if additionally the range of $T$ is some $L_{p}\left(\Omega, \mathbb{C}_{0}, P, B\right)$, where $\mathbb{C}_{0}$ is a sub- $\sigma$-field of $\mathbb{Q}(1<p<$ $\infty)$. The assumption on the range of $T$ is a very strong and unfavourable assumption which is unnecessary if $B=\mathbf{R}$ and $p \neq 2$. Assumptions on the range of the operator contradict the idea of characterization results. So it was the aim of all characterizations for conditional expectation operators, given in the real-valued

Received by the editors July 3, 1979 and, in revised form, March 26, 1980.

1980 Mathematics Subject Classification. Primary 60B11.

Key words and phrases. Conditional expectation, contractive operators, uniformly convex Banach spaces, strictly convex Banach spaces, $L_{p}$-spaces. 
case (see e.g. [1], [3], [5] or [7]) to use only algebraic and functional analytic properties (like (i)-(iv)) of the operator and to avoid assumptions on its range.

Our example shows that for each $p>1$ conditions (i)-(iv) do not characterize conditional expectation operators, even if $B$ is a (finite-dimensional) uniformly convex Banach space; this is in contrast to the case $B=\mathbf{R}$. If, however, $p=1$ and $B$ is a strictly convex Banach space we obtain the same result as in the real-valued case: Conditions (i)-(iv) characterize conditional expectation operators. We remark that our example also shows that this result is not true for arbitrary Banach spaces. Compared with Rao's result, there are the following differences:

(1) In the important case $p=1$, we can abandon Rao's assumption on the range of the operator.

(2) Rao assumed that the dual space $B^{*}$ is strictly convex. This is a stronger assumption than our assumption that $B$ is strictly convex (see $[2$, p. 212] or $[4, \mathrm{p}$. 245]).

(3) Rao proves a weaker result than stated. His proof works only under the additional assumption that $B^{*}$ has the Radon-Nikodym property with respect to $P / \mathscr{Q}_{0}$ : He uses $L_{1}\left(\Omega, \mathbb{Q}_{0}, P, B\right)^{*}=L_{\infty}\left(\Omega, \mathscr{Q}_{0}, P, B^{*}\right)$. This is according to Theorem 1 , p. 98, of [2] equivalent to the Radon-Nikodym property of $B^{*}$ with respect to $P / Q_{0}$, which is not implied by the strict convexity of $B^{*}$.

The methods used in this paper are completely different from the methods of M. M. Rao.

We remark that Rao's proof for the case $p>1$ has a gap: he uses Lemma 1 of his paper [8] which is endowed with an incorrect proof.

Example. Let $(\Omega, Q, P)$ be a probability space and consider the space $\mathbf{R}^{2}$ endowed with the norm $|x|=\left(\left|x_{1}\right|^{p}+\left|x_{2}\right|^{p}\right)^{1 / p}$, where $p>1$. Then we obtain a Banach space which we denote by $B_{p}$. The space $B_{p}$ is uniformly and hence strictly convex for $p>1$, whereas $B_{1}$ is not strictly convex. Let $Q_{1}, Q_{2}$ be two sub- $\sigma$-fields of $\mathbb{Q}$. Then the operator

$$
T: L_{p}\left(\Omega, Q, P, B_{p}\right) \rightarrow L_{p}\left(\Omega, \mathcal{Q}, P, B_{p}\right)
$$

defined by $T\left(X_{1}, X_{2}\right)=\left(P^{Q_{1}} X_{1}, P^{\mathbb{Q}_{2}} X_{2}\right)$ is obviously linear, idempotent and constant preserving. The operator $T$ is furthermore \|\|$_{p}$-contractive, since for each $X=\left(X_{1}, X_{2}\right) \in L_{p}\left(\Omega, Q, P, B_{p}\right)$ we have

$$
\|T X\|_{p}^{p}=P\left[\left|P^{Q_{1}} X_{1}\right|^{p}+\left|P^{Q_{2}} X_{2}\right|^{p}\right]<P\left[\left|X_{1}\right|^{p}+\left|X_{2}\right|^{p}\right]=\|X\|_{p}^{p}
$$

where the inequality follows from the convexity inequality for conditional expectations.

If $Q$ does not contain only sets of measure zero or one, then $T$ is not a conditional expectation operator, if we choose for instance $\mathbb{Q}_{1}=\{\varnothing, \Omega\}, \mathbb{Q}_{2}=\mathbb{Q}$.

TheOREM. Let $(\Omega, \mathcal{Q}, P)$ be a probability space and $B$ a strictly convex real Banach space. If $T: L_{1}(\Omega, Q, P, B) \rightarrow L_{1}(\Omega, Q, P, B)$ is an operator which is

(i) linear,

(ii) \|\|$_{1}$ contractive,

(iii) idempotent, 
(iv) constant preserving,

then there exists a sub- $\sigma$-field $\mathbb{Q}_{0} \subset \mathbb{Q}$ such that $T X=P^{\mathbb{Q}_{0}} X$ for all $X \in$ $L_{1}(\Omega, \mathcal{Q}, P, B)$.

Proof. We show at first that for all $b \in B(b \neq 0), A \in \mathbb{Q}$, there exists an $Q$-measurable and integrable function $f_{A, b}: \Omega \rightarrow \mathbf{R}$ with

$$
T\left(1_{A} b\right)=f_{A, b} b, \quad P \text {-a.e. }
$$

According to (i) and (iv) we have

$$
b=T b=T\left(1_{A} b\right)+T\left(1_{A} b\right)
$$

and hence (ii) implies

$$
\begin{aligned}
&|b|=|T b| \leqslant \int\left|T\left(1_{A} b\right)\right| d P+\int\left|T\left(1_{A} b\right)\right| d P \\
& \underset{\text { (ii) }}{\leqslant} \int\left|1_{A} b\right| d P+\int\left|1_{A} b\right| d P=|b|,
\end{aligned}
$$

whence

$$
|T b|=\left|T\left(1_{A} b\right)\right|+\left|T\left(1_{A} b\right)\right|, \quad P \text {-a.e. }
$$

As $B$ is strictly convex, (2) and (3) imply (1), where the functions $f_{A, b}$ can trivially be chosen $\mathcal{Q}$-measurable and integrable.

Let $b_{0} \neq 0$ and put $f_{A}:=f_{A, b_{0}}$. We shall show that

$$
T\left(1_{A} b\right)=f_{A} b, \quad P \text {-a.e. for all } b \in B \text {. }
$$

If $b=\lambda b_{0}$ for some $\lambda \in \mathbf{R}$, (4) trivially follows from (1) using that $T$ is homogeneous. Now let $b, b_{0}$ be linearly independent vectors of $B$. Then we obtain from (1), since $T$ is additive,

$$
f_{A} b_{0}+f_{A, b} b=f_{A, b+b_{0}}\left(b+b_{0}\right), \quad P \text {-a.e. }
$$

and hence

$$
\left(f_{A}-f_{A, b+b_{0}}\right) b_{0}=\left(f_{A, b+b_{0}}-f_{A, b}\right) b, \quad P \text {-a.e. }
$$

Consequently, as $b_{0}, b$ are linearly independent

$$
0=f_{A}-f_{A, b+b_{0}}=f_{A, b+b_{0}}-f_{A, b}, \quad P \text {-a.e. }
$$

i.e. $f_{A, b}=f_{A} P$-a.e. This implies (4). Since $T$ is linear and \|\|$_{1}$-continuous we obtain from (4) by simple arguments:

For each real-valued integrable function $g$ there exists a real-valued integrable function $\hat{T}(g)$ with

$$
T(g b)=\hat{T}(g) b, \quad P \text {-a.e. for all } b \in B \text {. }
$$

Therefore $\hat{T}: L_{1}(\Omega, \mathcal{Q}, P, \mathbf{R}) \rightarrow L_{1}(\Omega, \mathcal{Q}, P, \mathbf{R})$.

Since $T$ fulfills (i)-(iv) it is easy to see that $\hat{T}$ fulfills (i)-(iv) too. Hence according to the classical representation theorem (see Proposition I, 2.14 of [6]) there exists a sub- $\sigma$-field $\mathbb{Q}_{0} \subset \mathbb{Q}$ with $\hat{T}(g)=P^{\mathbb{Q}_{0}}$. Hence

$$
T(g b)=\left(P^{\mathbb{C}_{0}}\right) b=P^{\mathbb{Q}_{0}}(g b) \text { for all } g \in L_{1}(\Omega, \mathcal{Q}, P, \mathbf{R}) \text { and } b \in B \text {. }
$$

Since $T$ and $P^{\mathbb{Q}_{0}}$ are linear they coincide on all $Q$-simple functions and hence on $L_{1}(\Omega, \mathcal{Q}, P, B)$ too, because $T$ and $P^{\mathbb{A}_{0}}$ are \|\|$_{1}$-continuous. 


\section{REFERENCES}

1. R. R. Bahadur, Measurable subspaces and subalgebras, Proc. Amer. Math. Soc. 6 (1955), 565-570.

2. J. Diestel and J. J. Uhl, Vector measures, Math. Surveys, No. 15, Amer. Math. Soc., Providence, R.I., 1977.

3. R. G. Douglas, Contractive projections on an $L_{1}$ space, Pacific J. Math. 15 (1965), 443-462.

4. H. Heuser, Funktionalanalysis, Teubner, Stuttgart, 1975.

5. S. C. Moy, Characterizations of conditional expectation as transformation on function spaces, Pacific J. Math. 4 (1954), 47-63.

6. J. Neveu, Discrete parameter martingales, North-Holland., Amsterdam, 1975.

7. J. Pfanzagl, Characterizations of conditional expectations, Ann. Math. Statist. 38 (1967), 415-421.

8. M. M. Rao, Smoothness of Orlicz spaces, Indag. Math. 27 (1965), 670-690.

9. Inference in stochastic processes. III, Z. Wahrscheinlichkeitstheorie und Verw. Gebiete 8 (1967), 49-72.

Mathematisches Institut der Universität zu Köln, D-5000 Köln 41, Federal Republic of Germany

UNIVERStTÄt KONSTANZ, FAKUltÄt Für WirTSCHAFTSWISSENSChafTEN UND STATISTIK, D-7750 Konstanz, Federal Republic of Germany 